\title{
La movilidad académica internacional: experiencias de los estudiantes de Villavicencio, Colombia y Coatzacoalcos, México*
}

\author{
María Cristina Otero Gómez \\ Wilson Giraldo Pérez ${ }^{* * *}$ \\ José Luis Sánchez Leyva****
}

Recibido: 15 de enero de 2018

Evaluado: 4 de abril de 2018 Aceptado: 20 de mayo de 2018

\section{Resumen}

Este artículo presenta los resultados empíricos de un proyecto de extensión que tuvo como objetivo identificar y analizar las percepciones de 16 estudiantes de la Universidad de los Llanos de Colombia y de la Universidad Veracruzana de México acerca de su experiencia como participantes en un proceso de movilidad académica internacional. A partir de información recabada por medio de entrevistas semiestructuradas, se pudo establecer que el aspecto que tuvo mayor significado en su experiencia fue el contacto con otra cultura, y a nivel personal los jóvenes destacan la autonomía, la capacidad de adaptación y el trabajo en equipo.

Palabras clave: experiencia de los estudiantes, internacionalización, movilidad estudiantil.

Artículo de investigación: Se deriva de los resultados de un proyecto de proyección social fruto del convenio marco de cooperación internacional entre La Universidad de los Llanos de Colombia y la Universidad Veracruzana de México, liderado por el grupo de investigación Dinámicas de Consumo y por el Cuerpo Académico Investigación educativa, desarrollo y competitividad de las organizaciones y entidades económicas, de las respectivas universidades. Citar como: Otero, M, Giraldo, W y Sánchez, J. (2018). La movilidad académica internacional: experiencias de los estudiantes de Villavicencio, Colombia y Coatzacoalcos, México. Hallazgos, 15(30), 177-196. DOI: https://doi.org/10.15332/2422409X.4810

** Magíster en Mercadeo, Facultad de Ciencias Económicas, Universidad de los Llanos. ORCID: http://orcid.org/0000-0002-3241-7877. Contacto: motero@unillanos.edu.co.

*** Magíster en Mercadeo, Facultad de Ciencias Económicas, Universidad de los Llanos. ORCID: http://orcid.org/0000-0001-8514-9805. Contacto: wgiraldo@unillanos.edu.co.

**** Doctor en Gobierno y Administración Pública, Universidad Veracruzana campus Coatzacoalcos. ORCID: http://orcid.org./0000-0002-3519-0882. Contacto: luissanchez01@uv.mx. 


\section{The international academic mobility: students experiences from Villavicencio, Colombia and Coatzacoalcos, México}

\begin{abstract}
The following article introduces the empirical results from an extension project whose objective was to identify and analyze the perceptions of 16 students from the University of Los Llanos from Colombia and the Universidad Veracruzana from México, about their experiences as participants in an international Academic Mobility Process. Starting from collected data by means of structured interviews, we could infer that the most significant aspect in their experience was their contact with another culture, and at a personal level, young people outstand the autonomy, the capacity of adaptation and teamwork.
\end{abstract}

Keywords: the students experience, internationalization, student mobility.
Received: january 15, 2018

Evaluated: april 4, 2018

Accepted: may 20, 2018 


\section{A mobilidade acadêmica internacional dos estudantes de Villavicencio, Colômbia e Coatzacoalcos, México}

Recebido: 15 de janeiro de 2017

Avaliado: 4 de abril de 2018

Aceito: 20 de maio de 2018

\section{Resumo}

Este artigo apresenta os resultados empíricos de um projeto de extensão que teve como objetivo identificar e analisar as percepções de 16 estudantes da Universidade de los Llanos da Colômbia e da Universidade Veracruzana do México sobre a sua experiência como participantes em um processo de mobilidade acadêmica internacional. A partir da informação arrecadada por meio de entrevistas semiestruturadas, consegui-se estabelecer que o aspecto que teve maior significado na sua experiência foi o contato com outra cultura, e no âmbito pessoal os jovens destacam a autonomia, a capacidade de adaptação e o trabalho em equipe.

Palavras-chave: experiência dos estudantes, internacionalização, mobilidade estudantil. 


\section{INTRODUCCIÓN}

La internacionalización de la educación superior en el siglo xxi se ha convertido en uno de los pilares más importantes en el campo educativo, como respuesta a la apertura económica y diplomática de los países. Un aspecto que ha impulsado este proceso ha sido el cumplimiento de los factores de visibilidad nacional e internacional como uno de los criterios para la acreditación de instituciones y programas académicos (Consejo Nacional de Acreditación, 2013, p. 38), lo que conlleva al aumento en el número de convenios entre Instituciones de Educación Superior (IES) de diferentes países (Trejo y Rodrigues, 2017, p. 14). En consecuencia, las universidades se han visto obligadas a redefinir sus planes, objetivos, visión y funciones misionales para mejorar sus indicadores en materia de internacionalización e intercambio académico que permita el fortalecimiento de las IES en uno de sus quehaceres sustantivos: la docencia.

Dentro de las áreas estratégicas para la internacionalización de la educación superior se encuentra la movilidad estudiantil, encargada de promover el intercambio de experiencias interculturales y académicas. Según Lloyd (2016), quizás el área más visible de la internacionalización de la educación superior son los intercambios estudiantiles, cuya magnitud varía enormemente según la región y el país. La Organización de las Naciones Unidas para la Educación, la Ciencia y la Cultura (UnesCo) considera que las cifras para el incremento de la movilidad es muy favorable, se prevé que para el año 2025 la demanda de educación internacional llegará a 7.2 millones de estudiantes frente a los 1.2 millones reportados en el año 2000
(UnESCO, 2010). No obstante, estas migraciones corresponden principalmente a Estados Unidos como líder indiscutible en el número de estudiantes inscritos en sus instituciones con aproximadamente 1 millón al año para 2015; le siguen el Reino Unido, Francia y Australia (Lloyd, 2016). Por su parte, Colombia y México hacen su aporte a los flujos internacionales de movilidad estudiantil con 22.153 y 25.836 estudiantes respectivamente (UnESCO, 2012, p. 135). En este contexto, los países de América Latina, como receptores y como emisores, presentan una participación marginal en el panorama global y aunque tienen como preferencia de destino los Estados Unidos y algunos países europeos, han experimentado un interesante aumento en la movilización intrarregional (Bermúdez, 2015, p. 116).

La literatura disponible refleja la escasez en la información concerniente a los resultados de los procesos de internacionalización en Latinoamérica, puesto que las IES tienen aún una experiencia insuficiente en su seguimiento (Fresan, 2009, p.151). A pesar de las hondas repercusiones de la internacionalización, todavía es inadecuada su documentación en cuanto al funcionamiento y direccionalidad en las cadenas de movilidad, tanto en lo que concierne a los saberes como a sus portadores (Didou, 2016, p.5). Así mismo, el Instituto Internacional para la Educación Superior en América Latina y el Caribe revela que un problema en relación con los datos acerca de la movilidad estudiantil es que los países aplican criterios diferentes a la hora de aportar información sobre ese tipo de alumnos (IESLAC - UNesCo, 2009).

Sumado a lo anterior, Didou (2014) sostiene que las publicaciones sobre la internacionalización de las universidades de 
América Latina, en su mayoría, presentan los informes mediante indicadores más que en perspectivas cualitativas (p. 9). Por lo anterior, Rincón (2013) hace un llamado al requerimiento de superar el paradigma de los números en la movilidad internacional (p. 203). Esto demanda un interés en otros asuntos como es el caso de la valoración de las experiencias vividas, junto con el grado de satisfacción obtenido por los estudiantes participantes en los procesos de movilidad académica. Algunos investigadores han centrado su foco de interés en conocer las razones que motivan la decisión de realizar una estancia en un centro extranjero (Fernández, Pérez, y Vaquero, 2009). Otros se han apoyado en la estadística para establecer que las variables como las experiencias académicas y culturales en las universidades de acogida de estudiantes determinan la satisfacción general (Urquía y del Campo, 2016). Sin embargo, poco se sabe sobre aspectos cualitativos asociados a las apreciaciones de los estudiantes latinoamericanos una vez retornan a su país de origen; de allí que autores como Rienties, Hernández, Jindal-Snape y Alcott (2013) consideran que las experiencias académicas, culturales y de integración son determinantes en la percepción de la satisfacción de los estudiantes.

Visto lo anterior, es comprensible la urgencia de realizar un seguimiento a los resultados de los procesos de movilidad, puesto que ésta no puede verse como una situación episódica: debe consistir en un proceso permanente y continuo (Tünnermann, 2011, p. 96). En tal sentido, es importante avanzar en investigaciones que contribuyan en la documentación de la información sobre los análisis de los procesos de internacionalización regional.
Por lo expuesto anteriormente, este trabajo tuvo como objetivo identificar y analizar las percepciones de los estudiantes de la Universidad Veracruzana de México, campus Coatzacoalcos y de la Universidad de los Llanos de Colombia, sobre su experiencia como participantes en los procesos de movilidad internacional. Adicionalmente, se estudiaron las percepciones que tuvieron los alumnos en cuanto al nivel de preparación de su universidad, en comparación con la universidad de acogida.

El trabajo se estructura en tres partes, en la parte inicial se presenta el contenido conceptual centrado en la revisión de literatura. La siguiente sección explica la metodología del estudio cualitativo y describe los hallazgos e interpretaciones de la investigación. Por último, se discuten las principales limitaciones del estudio y se sugieren futuras líneas de investigación.

\section{Marco de Referencia}

\section{La movilidad estudiantil}

Existe riqueza en cuanto a las investigaciones relacionadas sobre movilidad académica internacional en la educación superior (Rodríguez, 2005; Brooks y Waters, 2011; García, 2013; Morales y Manosalba, 2016; Hercog y Van, 2017). Algunos autores han demostrado mediante estudios empíricos avances concretos en materia de movilidad estudiantil, cuya orientación se ha enmarcado en temas que invitan a reflexionar críticamente sobre el significado y las implicaciones de la movilidad internacional con fines educativos y su relación con una variedad de desigualdades, a nivel macro, meso y micro (Bilecen y Van Mol, 2017, p. 1243). 
Otros estudios revelan el significado de la movilidad obedeciendo a interpretaciones sociales adaptadas a determinadas culturas, por ejemplo, en regiones de Asia la educación en el extranjero de un estudiante se interpreta como un medio de ascenso social para toda la familia, lo que conlleva a la movilidad social y al avance profesional entre dichas familias (Huang y Yeoh, 2005; Findlay, King, Smith, Geddes y Skeldon, 2012). Así mismo, en India los determinantes de la movilidad internacional están soportados en el tipo de universidad, en el campo de estudio, en la proyección profesional y laboral; y en el apoyo moral y financiero por parte de la familia (Hercog y Van, 2017). De otro lado, en un estudio realizado en Turquía se pudo establecer que las razones que influyen en la movilidad de estudiantes extranjeros procedentes de países vecinos hacia este país, está mediada principalmente por la calidad académica, por encima de aspectos significativos como aspiraciones laborales, proximidad geográfica y racionalidad económica (Kondakci, 2011).

En particular, la investigación en el contexto español muestra resultados acerca de la influencia del trabajo en equipo y su efecto en el aprendizaje colaborativo mediante la vinculación de estudiantes extranjeros y nacionales (Rienties, Hernández, Jindal-Snape y Alcott, 2013). Otra investigación destaca que el género de los estudiantes desempeña un papel importante en la elección de la universidad anfitriona junto con el interés de desarrollar competencias interculturales y lingüísticas en el país de destino (Urquía y del Campo, 2016).

A nivel de Latinoamérica, la investigación de Trejo y Rodrigues (2017) se centra en el análisis de la movilidad estudiantil como una forma de acceder a la nacionalidad española por parte de brasileños, mexicanos y colombianos. Asimismo, Geldres, Vásquez, y Ramos (2013) presentan una caracterización en términos de destino, origen, programa académico y tasa neta de movilidad académica en una universidad de Chile.

Como se observa, en la literatura disponible se identifica un relativo consenso en determinar que la movilidad estudiantil se analiza a partir de las razones que motivan el desplazamiento desde el país de origen. Dichas investigaciones guardan relación con la definición general de movilidad académica que consistente en:

Un proceso que implica el desplazamiento físico de una persona desde su alma máter hacia otra institución con el propósito de realizar una actividad académica que complemente su conocimiento, formación o actividades de investigación. Esta estrategia tiene como objetivo propiciar encuentros pedagógicos, desarrollar competencias multiculturales, habilidades lingüísticas, fortalecer el sentido de identidad y desarrollar otras competencias necesarias para la interacción en la globalización. (Ministerio de Educación Nacional, 2015, p. 15).

En el caso de México, la internacionalización de la educación superior se define desde el Plan Nacional de Desarrollo. En él, se plasman las líneas de acción que permitirán el intercambio de experiencias en contextos internacionales a través de líneas de acción conducentes a: "impulsar programas de posgrado conjuntos con instituciones extranjeras de educación superior en áreas 
prioritarias para el país y crear un programa de estadías de estudiantes y profesores en instituciones extranjeras de educación superior" (Plan Nacional de Desarrollo 2013-2018, pp. 123-124). Estas líneas de acción pretenden que los planes y programas de estudio sean pertinentes y contribuyan para que los estudiantes avancen de manera exitosa en su formación profesional en la que desarrollen aprendizajes significativos y competencias que les permitan el acceso a un mercado laboral dinámico, global y cambiante.

Particularmente, para la Universidad Veracruzana, la movilidad estudiantil expresada a través de la Dirección General de Relaciones Internacionales, es definida como:

Una estrategia institucional que promueve que los estudiantes de licenciatura y posgrado realicen estancias cortas o semestrales en otras universidades nacionales e internacionales con la finalidad de cursar experiencias educativas, llevar a cabo acciones de investigación, realizar prácticas profesionales o cualquier otra actividad que implique reconocimiento curricular. (Dirección General de Relaciones Internacionales, 2013).

Asimismo, coincide con las investigaciones presentadas previamente, en el sentido de mostrar beneficios implícitos como: la experiencia académica en otros espacios educativos, culturales y sociales; desarrollo de una mejor comprensión de las realidades diferentes a la propia; y la creación de redes de estudiantes y amistades con otros jóvenes de diversas partes del mundo, entre otros (Dirección General de Relaciones Internacionales, 2013).
No obstante, este proceso debe llevar implícito un seguimiento que permita conocer las impresiones de los propios beneficiarios, puesto que las experiencias adquiridas por los estudiantes son un insumo valioso a la hora de evaluar la situación de los programas académicos institucionales, su pertinencia y su abordaje respecto a los socios internacionales (Ministerio de Educación Nacional, 2015, p. 33). En este sentido, conocer la experiencia de los participantes en programas de intercambio resulta primordial pues no solamente se conocen las motivaciones que los empujaron a realizar una estancia en otro centro educativo, sino que permite conocer el cumplimiento de sus expectativas una vez que retornan a su universidad de origen. Así pues, las experiencias de cada individuo son un insumo invaluable y muchas veces no se generan espacios para que ellos puedan contribuir también en la promoción de la movilidad (MEN, 2015, p. 49). El presente artículo intenta llenar este vacío de investigación utilizando una técnica cualitativa la cual se describe en el siguiente apartado.

\section{Metodología}

\section{Contexto del estudio}

La investigación se desarrolló con estudiantes de dos universidades públicas que corresponden a ciudades con características similares en cuanto a tamaño, nivel de desarrollo, condiciones sociales y algunos patrones culturales ligados a la estructura familiar y al modelo de crianza de sus integrantes. La razón por la cual se seleccionaron los estudiantes de las ciencias empresariales y de la administración para este estudio están soportadas en las tendencias 
de la región de América Latina y el Caribe, donde casi uno de cada cuatro alumnos trashumantes estaba matriculado en una carrera afín a estas ciencias (IESLAC - UNESCO, 2009). También porque son temáticas de interés claramente definidas por los objetivos estratégicos y geopolíticos de inserción económica internacional y de apertura de mercados (Morales y Manosalba, 2016, p. 29).

\section{Los informantes}

Los participantes del estudio fueron 16 estudiantes, de ellos diez de sexo femenino y seis de sexo masculino. Para el caso de Villavicencio, la edad de los estudiantes oscila entre los 23 y 25 años, cursantes de los últimos semestres de formación profesional; pertenecientes a los niveles medio y bajo de la escala social. Para el caso de Coatzacoalcos, la edad de los estudiantes oscila entre los 21 y 27 años. De ellos, siete se encuentran cursando los últimos semestres de su formación profesional, mientras que los tres restantes cursan un posgrado en la Facultad.

Se realizaron dos sesiones de entrevistas, la primera desarrollada de forma grupal en septiembre de 2016 y la segunda desarrollada individualmente en junio de 2017, la duración fue alrededor de una hora y media cada una. En la primera sesión participaron cuatro estudiantes - mujeres - de Coatzacoalcos y cuatro estudiantes de Villavicencio - dos hombres y dos mujeres-. En la segunda sesión participaron seis estudiantes de Coatzacoalcos - tres hombres y tres mujeres - y dos de Villavicencio - un hombre y una mujer - .

La experiencia vivida tiene dos matices, por un lado, se describen las vivencias de los estudiantes de Coatzacoalcos en la
Universidad de los Llanos en Villavicencio; por otro, se detallan las vivencias de los estudiantes de la Universidad de los Llanos en la Universidad Veracruzana campus Coatzacoalcos, en la Universidad de Guadalajara, en la Universidad de Chapingo y en la Universidad Nacional Autónoma de México.

Los informantes de Villavicencio experimentaron la movilidad académica internacional bajo la modalidad de intercambio académico durante un semestre de formación universitaria en México. Por su parte, los informantes de Coatzacoalcos tuvieron su experiencia bajo la modalidad de misión, entendida como "viajes académicos cortos con el propósito de permitir al estudiante tener un acercamiento con diferentes instituciones [...] permite ampliar la visión internacional de los estudiantes por el contacto que alcanzan a tener a nivel académico, científico, político, cultural y económico del país que visitan" (MEN, 2015, p. 30).

\section{Proceso de recogida de datos}

Se empleó una metodología de carácter cualitativo utilizando como técnica de investigación la entrevista semiestructurada. El uso de esta técnica se debe a que es particularmente útil para explorar los conocimientos y experiencias de las personas en un ambiente de interacción, permite examinar lo que la persona piensa, cómo piensa y por qué piensa de esa manera (Hamui y Varela, 2013). En cuanto a los aspectos éticos, a los participantes se les informó que el tratamiento de los datos es anónimo y confidencial, y durante la sesión tenían la libertad de abandonar el estudio cuando lo requirieran.

De este modo se inició la generación de los datos, donde los investigadores utilizaron 
un protocolo de entrevista semiestructurada basado en las preguntas abiertas de un estudio previo realizado por Fresan (2009) y adaptado para dar cumplimiento al objetivo propuesto en la presente investigación. De igual forma, se consideraron los lineamientos del Ministerio de Educación Nacional de Colombia para evaluar los resultados de la movilidad internacional, asociados al idioma, las competencias multiculturales, el trabajo en contextos interculturales, la autoconfianza, el liderazgo, el aspecto financiero, desempeño académico, apoyo financiero, recepción por parte de la universidad anfitriona, entre otros (MEN, 2015, pp. 21-27).

Todas las entrevistas fueron grabadas en video y audio; una vez finalizaron las sesiones se procedió a su transcripción. Los resultados obtenidos se procesaron a través del software AtlasTi. Se analizaron las respuestas de los participantes, con el fin de formar un concepto general y una primera comprensión del fenómeno global, así como para detectar acuerdos y desacuerdos significativos entre los participantes de los dos países.

Al finalizar la entrevista se solicitó a los informantes que evaluaran ocho aspectos relacionados con temas académicos y de cultura ciudadana, comparando su universidad de origen frente a la universidad receptora.

\section{Hallazgos}

La tabla 1 presenta una síntesis de los resultados a partir del discurso oral de los participantes del estudio. Estos hallazgos corresponden a la representación de la movilidad académica consignada en una sola palabra.
Tabla 1. Representación de la movilidad académica consignada en una sola palabra.

\begin{tabular}{ll}
\hline \multicolumn{1}{c}{$\begin{array}{c}\text { Estudiantes de } \\
\text { Coatzacoalcos (M) }\end{array}$} & \multicolumn{1}{c}{$\begin{array}{c}\text { Estudiantes de } \\
\text { Villavicencio (C) }\end{array}$} \\
\hline Experiencia & Experiencia \\
Crecimiento & Interesante \\
Increíble & Aprendizaje \\
Enriquecedora & \\
Éxito & \\
Inolvidable & \\
\hline
\end{tabular}

Fuente: elaboración propia

\section{Aportes de la movilidad académica internacional}

La movilidad estudiantil es un proceso inherente a los cambios en el ser humano, por lo cual, estos jóvenes tienen una perspectiva diferente de la vida cuando retornan a su lugar de origen. En este contexto, cuando los participantes son indagados sobre los aspectos que cambiaron en su vida a partir de la movilidad, algunos señalan que los principales cambios corresponden a mejorar la convivencia, ser más autónomos, aumentar su autoconfianza y administrar de forma eficiente sus recursos financieros. Relativo a lo anterior algunos señalaron: “Me permitió aprender a administrar mis recursos y saber cómo hacer uso de ellos. Por otro lado, pude relacionarme con más personas, adaptarme a las distintas formas de pensar y a trabajar en equipo" (E6 [10:02]) y “Aprendí a ser cauteloso en el uso de mis recursos económicos (E14 [11:12]). Asimismo, otros de los entrevistados afirman: "La convivencia fue la parte más importante, las relaciones humanas y el intercambio de culturas me han brindado una nueva forma de ver la vida" (E8 [10:12]). Este proceso también sirvió para aprender a valorar aspectos de la cotidianidad: "Lo que aprendí de este viaje es que por más lejos que uno vaya y por más 
que uno conozca, no hay nada como su tierra, su gente, sus costumbres y sobre todo como su comida" (E10 [10:14]). Desde este punto de vista, los entrevistados encontraron que la movilidad no sólo contribuye en su formación profesional, sino que gracias a la experiencia vivida les genera grandes aportes como seres humanos.

\section{Recepción por parte de la universidad anfitriona}

El recibimiento de los visitantes es crucial puesto que se trata de la imagen que construyen sobre la universidad que los acoge. Adicionalmente, sirve de espacio para facilitar el acercamiento entre los profesores, los directivos y demás estudiantes tanto internos como aquellos que también están en condición de movilidad entrante.

Por ello, se indagó sobre la percepción en relación con la actitud de aceptación de la comunidad académica al llegar a la institución de destino. Al respecto, dos de los entrevistados de México plantean lo siguiente: "El trato que nos dieron al ingresar a sus instalaciones fue excelente, todos los profesores e incluso los estudiantes nos trataron de una manera muy amable" (E1 [13:17]) y "En lo personal hice amistades y vínculos con los maestros investigadores, puedo asegurar una relación de trabajos colaborativos y hasta poder regresar como ponente en sus congresos y revistas de investigación adscrita a la Facultad de Ciencias Económicas" (E8 [12:11]). Por su parte, la experiencia de una estudiante colombiana se describe como: "Puedo decir que su apoyo y hospitalidad no pudo ser mejor, me siento agradecida y afortunada de haber elegido a la universidad de Guadalajara como destino" (E11 [10:17]). En términos generales todos coincidieron en rescatar la amabilidad, el respeto y el hecho de sentirse como en casa.

En contraste con el consenso en las respuestas favorables respecto del trato personal, las opiniones cambiaron cuando se les preguntó sobre su percepción en cuanto al nivel de preparación de la institución receptora. Las respuestas de los mexicanos presentaron variaciones debido a que fueron recibidos por grupos diferentes en las visitas que realizaron en el año 2016 y 2017. Así, para el primer año un informante manifiesta: "La Universidad de los Llanos se encuentra bien equipada, aunque hay cosas que puede mejorar; en cuanto al conocimiento y la participación de los alumnos se refleja la buena calidad docente (E1 [11:05]). En el segundo año un informante hace la siguiente descripción: "En cuestiones de planeación algunos profesores y estudiantes no llegaban puntuales a la hora de realizar la actividad con ellos, su infraestructura es un poco mala al no contar con los equipos necesarios en las aulas de clases" (E5 [9:14]); "Considero que existe deficiencia en cuanto a la logística y la planeación, realmente no hubo una organización previa a nuestra llegada" (E6 [13:12]). Los jóvenes de México también reconocieron aspectos positivos como: "El nivel de los profesores y estudiantes es muy bueno, ya que pese a las deficiencias se tiene un amplio conocimiento y participación activa" (E8 [14:02]) $\mathrm{y}$ "Los maestros son personas muy apasionados por sus carreras, casi todos siguen estudiando algo; les gusta mucho investigar y motivan a sus alumnos a hacerlo. Les enseñan a expresarse en público como parte de su formación integral; no escuché a ningún alumno al momento de dar una opinión titubear o emitir frases incoherentes. A pesar 
de la pronta creación de las carreras, ellos ya celebran varias ediciones de sus revistas" (E10 [12:07]). Por su parte, las respuestas de los colombianos fueron: “¡Excelente! las condiciones de la Universidad de Guadalajara fueron las adecuadas desde el primer a último día" (E11 [12:05]); otro informante contó su percepción sobre la Universidad Veracruzana "La infraestructura posee falencias pero están trabajando para superarlas" (E12 [9:12]). "La Universidad de Chapingo tiene dispuestos dormitorios para los jóvenes de intercambio, eso es fundamental para acomodarse a la vida en otro país, aunque son espacios pequeños, se facilita todo" (E15 [12:11]). “La recepción por parte de la UNAM es impresionante, es aquel momento donde te encuentras con personas provenientes de muchos países, escuchas diferentes idiomas, y empiezas a entender lo global del mundo académico" (E16 [8:13]). En este escenario es posible identificar que para los jóvenes, a pesar de las falencias que puedan presentarse en la planta física y en el equipamiento, existen otros elementos como la calidad del docente, los protocolos de recepción y el desempeño de los estudiantes, que inciden en la calidad percibida y en la satisfacción durante su experiencia.

\section{Percepción sobre la ciudad de destino}

Asociado al punto anterior, las creencias sobre un lugar influyen en la construcción de imágenes o estereotipos que motivan o desestimulan la decisión de viajar. Para el caso, se trata de dos ciudades pertenecientes a países que en las últimas décadas se han visto afectados principalmente por conflictos del orden social, acompañado de crisis políticas y económicas. En consecuencia, los participantes tenían una expectativa que cambió cuando vivieron la experiencia de encontrarse en el destino de movilidad. Así lo manifiesta uno de los informantes de México: "Antes de conocer Villavicencio mis expectativas se centraban en lo que dicen los medios internacionales, tenía muchas ganas de conocer aquel país pero debo admitir que tenía cierto temor de ser afectada por el crimen organizado, que los medios se encargan de recalcar tanto; sin embargo desde que arribamos a Villavicencio me sentí como en casa, la ciudad es muy tranquila y las personas muy amables, sin duda el destino superó mis expectativas de forma positiva" (E1 [13:15]). En la misma línea, otro entrevistado de Colombia relata su experiencia. “Mi mamá todo el tiempo estuvo preocupada por mi estadía en México, me decía que no saliera de la Universidad porque solo allí podía estar segura, debido a las noticias negativas que transmiten, pero una vez instalada allá uno se da cuenta que la realidad es otra, hay muy buenas personas, es muy bonito y al igual que en Colombia uno debe tener ciertas prevenciones" (E13 [12:13]). Estas percepciones permiten reconocer que los medios de comunicación se encargan de distorsionar las características reales que tiene un destino académico, la mayoría de estudiantes poseían creencias que les generaba temor al estar lejos de sus hogares.

La movilidad académica permite, entre otros aspectos, el desarrollo de competencias multiculturales y el fortalecimiento del sentido de identidad. De este modo, se indagó sobre el aporte de esta experiencia al enriquecimiento cultural. Esta perspectiva es ilustrada en el siguiente segmento de entrevista: “De Villavicencio aprendí mucho acerca de su cultura, de sus costumbres que 
son muy distintas a las nuestras, pero que están igual de arraigadas en sus habitantes desde las comidas y bailes típicos, sus tradiciones, sus formas de pensar, hasta parte de su historia. Todo eso que aprendí abrió por completo mi mente y aumentó mis ganas de superarme para salir y conocer más lugares, otras culturas" (E2 [20:13]). “Durante mi estancia pude conocer ciertos hábitos que tienen los habitantes de Villavicencio, como el hecho de lo temprano que comienza su día y de los activos que son, que son ricos en gastronomía y paisajes. Algo que me agradó y sorprendió mucho, fue notar como la mayoría de los habitantes conoce la historia o aspectos de su ciudad y de su país; datos que cada habitante debe conocer sobre su país, pero en que en mi caso no conozco" (E3 [14:13]). "Los estudiantes de la universidad de los Llanos nos enseñaron el significado de la identidad cultural, ellos aman y respetan su tierra, se enorgullecen de ella a pesar de las dificultades por las que puedan atravesar, es muy bonito ver como conservan sus tradiciones y practican sus danzas típicas" (E1 [20:13]). Por su parte, un informante colombiano se identifica con estas apreciaciones: "Más que un enriquecimiento cognitivo, fue cultural, escuchar un acento diferente, una manera distinta de expresar ideas, actividades diferentes al momento de divertirse, y su variada y rica gastronomía" (E13 [43:03]). En función de lo manifestado por los entrevistados, la mayoría afirmó que el hecho de convivir con otra cultura hace que cambie su forma de pensar, puesto que no es lo mismo leer, ver o escuchar algo acerca de otro país, a vivir la experiencia de estar allí.

\section{Valoración de la experiencia}

Entre los resultados más llamativos de estas experiencias se hace especial énfasis en los aspectos positivos, en los conocimientos y habilidades extra académicos adquiridos durante la estancia internacional y en el apoyo otorgado por parte de la universidad emisora para la movilidad académica.

De este modo, existe un marcado acuerdo en que el enriquecimiento cultural y académico fue lo más significativo $\mathrm{y}$, a la vez, se convierte en un generador de nuevas expectativas. Un entrevistado de México manifiesta: "Esta estancia me ha motivado a hacer investigación, a publicar en revistas internacionales, y en un futuro no muy lejano poder estudiar un doctorado" (E8 [49:07]). En cuanto a los aspectos negativos, los jóvenes consideraron que la experiencia no podría tener esa clase de calificativos, por el contrario vieron en las situaciones de frustración una oportunidad para afrontar nuevos retos.

Aparte del aprendizaje académico, los entrevistados manifestaron que algunas situaciones cotidianas les generaron mucha tensión, por ejemplo, un entrevistado manifestó lo siguiente: "La habilidad para manejar una moneda distinta al peso mexicano, sin duda fue muy difícil al principio acostumbrarse a la moneda de Colombia" (E5 [28:17]). Por el contrario aspectos asociados a la diversión se convirtieron en aprendizaje, así lo ratificaron dos de los informantes: "En México aprendí nuevos bailes, y una nueva forma de diversión en la feria de Aguascalientes" (E16 [32:07]) y “Además tuve la oportunidad 
de realizar excursiones en donde conocí distintos lugares representativos y turísticos de la zona de Veracruz" (E12 [28:14]).

Un componente importante que brinda esta experiencia es poder analizar el grado de apoyo financiero y emocional por parte de las directivas de las universidades emisoras. En este sentido, cuando se consulta a los entrevistados sobre las acciones realizadas por su universidad para facilitar el desplazamiento, ellos revelan la existencia de grandes diferencias. Lo anterior se evidencia a través del discurso de varios participantes, por un lado, los colombianos manifiestan: "La Unillanos solamente me entregó una carta de presentación dirigida a la universidad de intercambio" (E15 [50:21]); "La Unillanos me brindó un apoyo económico con el cual cubrí mis gastos de tiquete aéreo y aunque su seguimiento no fue mucho, fue la que me permitió vivir la experiencia" (E11 [35:18]); "La Universidad de los Llanos me apoyó de forma económica con media beca; y también realizó los trámites y papeleos en tiempo y forma necesarios para este proceso" (E12 [46:20]). Por su parte, los entrevistados de la Universidad Veracruzana parecen indicar un punto de vista opuesto: “El apoyo otorgado por parte de la Universidad Veracruzana para la movilidad académica asciende a MXN45.000.00" (E5 [47:22]); “Recibí en un $100 \%$ el apoyo de la universidad Veracruzana. Gracias a esto se pudieron solventar todos los gastos durante la estancia" (E6 [53:18]); “Considero que el apoyo por parte de la universidad es muy importante y de mucha utilidad" (E7 [26:05]) y "Amo mi facultad, cumple con todas mis expectativas como Universidad. Si tuviera que evaluar el apoyo en escala del 1 al 5, mi calificación seria 5" (E8 [56:12]. En función del discurso de los entrevistados mexicanos, las percepciones que ellos poseen asignarían a las directivas un rol protagónico en el éxito de la movilidad internacional.

\section{Valoración de la satisfacción}

La satisfacción es un concepto que se relaciona con lo que un individuo desea y con el resultado que espera, por tanto, se trata de un fenómeno subjetivo que se concreta posterior a la experiencia. En la mayoría de respuestas que brindaron los entrevistados en torno a la valoración general de la estancia internacional hubo un acuerdo en sus respuestas: "Excelente" (E4 [35:16]); "Enriquecedora" (E7 [46:18]); “Adecuada" (E9 [21:06]) y "Muy buena" (E14 [41:12]). Igual sucedió con la pregunta ¿recomendaría a la universidad de destino para intercambios académicos?, donde la respuesta de la totalidad de participantes fue "si". No sucedió lo mismo con la pregunta ¿le gustaría volver a la universidad de destino bajo la modalidad de intercambio académico?, en este caso se trató de la Universidad de los Llanos como receptora, donde un entrevistado manifestó: "Si tuviera alguna otra oportunidad de movilidad optaría por conocer un lugar distinto" (E7 [49:26]). Los quince entrevistados restantes manifestaron un "si" rotundo respaldando sus respuestas basados en los aspectos académicos, en la hospitalidad de los residentes y en la oportunidad de conocer más de la cultura local, entre otros. Al respecto, se puede inferir que la satisfacción del estudiante inmerso en una experiencia de movilidad académica internacional está mediada por diversos factores: académicos, culturales, personales, sociales e institucionales. 


\section{Comparación del nivel de preparación de la universidad receptora frente a la universidad emisora}

La experiencia adquirida por lo estudiantes a través de la movilidad internacional, entre otros aspectos, permite la emisión de juicios y la realización de comparaciones entre lo que brinda su universidad y lo que ofrece la institución receptora. De este modo, al finalizar la entrevista se pidió a los jóvenes que realizaran una evaluación. La tabla 2 presenta el promedio de las valoraciones asignadas a cada variable, donde 1 representa que la universidad receptora es mucho mejor en comparación con la universidad emisora; y 5 que la universidad receptora es muy inferior frente a la universidad emisora.

Los resultados expuestos permiten inferir que los entrevistados mexicanos consideran que la Universidad de los Llanos es mejor que la Universidad Veracruzana en aspectos como: la cultura científica, la comunicación, la ciudadanía y los conocimientos e intereses por la carrera elegida. Así mismo, sienten que están en condiciones similares en aspectos como: el pensamiento matemático, la capacidad para el aprendizaje autodirigido y en el trabajo en equipo. También consideran que sus destrezas tecnológicas son superiores en comparación con la Universidad de los Llanos.

En cuanto al análisis de la percepción de preparación de las universidades mexicanas objeto de estudio, en comparación con la Universidad de los Llanos, se puede afirmar que los estudiantes consideran que existen mejores condiciones en materia ciudadana. También destacan las destrezas tecnológicas que poseen los estudiantes mexicanos. Aseguran que la variable comunicación en otra lengua internacional se encuentra mejor valorada, concretamente en

Tabla 2. Percepción del nivel de preparación de las universidades receptoras versus emisoras.

\begin{tabular}{lll}
\hline Variables evaluativas & $\begin{array}{c}\text { Valoración de los es- } \\
\text { tudiantes mexicanos }\end{array}$ & $\begin{array}{c}\text { Valoración de los estu- } \\
\text { diantes colombianos }\end{array}$ \\
\cline { 2 - 3 } & $\begin{array}{c}\text { Unillanos / } \\
\text { Uveracruzana }\end{array}$ & $\begin{array}{c}\text { Universidades mexica- } \\
\text { nas / Unillanos }\end{array}$ \\
\hline Comunicación en lengua materna y en otra lengua & 2,8 & 2,2 \\
internacional & 3,1 & 3,0 \\
\hline Pensamiento matemático & 2,8 & 3,2 \\
\hline Cultura científica & 2,8 & 2,0 \\
\hline Ciudadanía & 3,8 & 2,5 \\
\hline Destrezas tecnológicas & 3,1 & 3,8 \\
\hline Capacidad para el aprendizaje auto-dirigido & 3,1 & 3,2 \\
\hline Capacidad para la socialización y el trabajo en equipo & 2,7 & 3,0 \\
\hline Conocimientos e intereses por la carrera elegida & & \\
\hline
\end{tabular}

1= Mucho mejor; 2 = Mejor; 3 = Igual; 4 = Inferior y 5 = Muy inferior

Fuente: elaboración propia 
la Universidad Nacional Autónoma de México, donde alcanzan la máxima puntuación debido a que existen profesores bilingües y estudiantes europeos de habla inglesa.

En la variable pensamiento matemático coinciden con los estudiantes mexicanos al afirmar que se encuentran en igualdad de condiciones. Además perciben que existe paridad en los conocimientos e intereses por la carrera elegida que tienen los estudiantes.

Finalmente, perciben como menos favorables las condiciones en México para las variables de capacidad para la socialización y el trabajo en equipo, la cultura científica y especialmente la capacidad para el trabajo autodirigido. En esta última variable los estudiantes colombianos afirman sentir que tienen un mejor dominio de su tiempo de estudio independiente.

\section{Discusión}

Al revisar los hallazgos obtenidos en esta investigación, se identificaron varios aspectos que contribuyen a la comprensión de los procesos de movilidad académica, desde una visión cualitativa. En este marco se discuten dos aspectos: la experiencia desde la perspectiva del estudiante y también la experiencia asociada al apoyo de las universidades en los procesos de movilidad internacional.

La primera visión ubica a los estudiantes de los dos países en un escenario placentero, gracias al trato personal de los profesores y estudiantes de la universidad y de la ciudad de acogida. Este comportamiento tiene sustento en los aportes de Basabe, Zlobina y Páez (2004) quienes argumentan que los países de Latinoamérica tienden a pertenecer a culturas colectivistas que funcionan grupalmente (p. 127). Estos resultados se encuentran en consonancia con Castro y Lupano (2013) toda vez que los nativos valoran el compartir actividades grupales, esto puede favorecer la integración de los inmigrantes (p. 275). Por el contrario, difiere de un estudio donde se identificaron experiencias negativas como discriminación, exclusión y bullying por parte de profesores y estudiantes al considerar que los extranjeros sólo van para quitarles el dinero (Navarrete, Mayor, Rangel, González, Zavala y Guillén, 2015, p. 22).

Sin duda alguna, otro aspecto importante resultado de la experiencia de los jóvenes es que la movilidad genera emociones encontradas. Por un lado, está presente el deseo de conocer culturas diferentes a la de su país de origen y del país que están visitando; lo que es coherente con la afirmación de Fernández, Carreño, Cea, Santander y Yáñez (2016), debido a que los estudiantes consideran que tener experiencias de este tipo, aumenta el deseo de viajar, ampliar horizontes y conocer una cultura a través de la inmersión (p. 4). Por otro lado, se genera un sentimiento de nostalgia hacia su cultura, de este modo, añoran reencontrarse con sus familias y sus tradiciones, sumado a un patriotismo que los enorgullece de su origen. Este comportamiento podría atribuirse a factores demográficos como la edad y el estado civil. Fiocch y Rojas (2015) a partir de su investigación, manifiestan que pareciera ser que las personas que participan en programas de intercambio en edades muy tempranas son menos apegadas a sus raíces y tradiciones (p. 229). Recordemos que la edad de los entrevistados oscila entre los 21 y 27 años, por tanto, podría considerarse 
que a nivel general el sentimiento de apego a su tierra es mayor.

Desde otra perspectiva, a partir del análisis de los aspectos institucionales y su incidencia en el grado de satisfacción del estudiante, conviene señalar grandes diferencias presentadas entre la Universidad de los Llanos y la Universidad Veracruzana. En primer lugar, diversas investigaciones coinciden en afirmar que la principal barrera percibida por los estudiantes para participar en un proceso de movilidad responde a la falta de apoyo financiero recibido de su universidad (Fernández, Fernández y Vaquero, 2007; Bilecen y Van Mol, 2017). Desde esta perspectiva y partir de las respuestas de los entrevistados, podría afirmarse que existen grandes brechas en la asignación de recursos. En el caso de la Universidad de los Llanos, los informantes manifestaron que durante el año 2016 no recibieron apoyo económico y en la movilidad de 2017 cada estudiante recibió un único desembolso equivalente a USD 1.167 para ser distribuidos durante todo el semestre de intercambio. Por el contrario, en la Universidad Veracruzana durante la misión realizada en el año 2016 por un periodo de dos semanas, cada estudiante recibió la suma de USD 1.962; y en la misión de 2017, cuya duración también fue de dos semanas, cada estudiante recibió USD 2.522. Por tanto, los resultados arrojados en relación con la Universidad Veracruzana no coinciden con las investigaciones citadas.

Ahora bien, se debe reconocer que son facultades que están experimentando las primeras experiencias en los procesos de movilidad académica, y tal como afirma Fresan (2009): “En una institución cuyos actores no tienen experiencia en [...] el programa de
Movilidad, el inicio necesariamente conlleva una evaluación negativa de la calidad del apoyo administrativo a este proceso" (p. 151). Aunque este resultado no se ajusta a la experiencia de la Universidad Veracruzana, se espera que a medida en que se destinen más recursos, como es el caso de la Universidad de los Llanos, y a medida en que sean más frecuentes los desplazamientos internacionales, podrá mejorarse el desempeño tanto en la modalidad entrante como saliente.

De esta manera, salvo por el respaldo institucional, se puede afirmar que en términos generales, los resultados de la experiencia vivida por el total de entrevistados fue muy similar. Esto difiere con los resultados del estudio realizado por Trejo y Rodrigues (2017), quienes a partir de los análisis de las experiencias de movilidad en estudiante mexicanos, colombianos y brasileños en España, identificaron que la experiencia vivida por los diferentes migrantes no es experimentada de la misma manera (p. 18).

\section{Conclusiones}

En los estudiantes que realizan procesos de movilidad académica internacional, la experiencia que viven les permite afirman que sus vidas cambian, puesto que desarrollan habilidades que hasta ese momento creían no tener. También adquieren una mayor responsabilidad en el desarrollo de sus actividades, especialmente en las financieras.

A nivel de países, se puede inferir que los estudiantes de la Universidad de los Llanos presentan mayores dificultades para el apoyo financiero brindado desde la oficina encargada de la internacionalización 
académica, sin que esto llegue a convertirse en un obstáculo para realizar su movilidad. Caso contrario presentan sus pares mexicanos a quienes su universidad les facilita mayor apoyo financiero para el desarrollo de su misión académica, la cual se realiza con el acompañamiento de profesores. Esto evidencia que el apoyo financiero es el aspecto que presenta mayores diferencias entre las dos universidades.

Frente a las percepciones de recepción, los estudiantes manifiestan que son las personas - estudiantes, profesores y residentesquienes compensan las dificultades que se pueden presentar a nivel de baja acogida directiva. Se deben hacer entonces mayores esfuerzos por acercar a los directivos de las universidades con los estudiantes entrantes.

Aspectos como la cultura expresada en la gastronomía y sus bailes, junto con los atractivos naturales de cada ciudad, además de las oportunidades académicas para avanzar en la formación posgradual, así como la motivación de vincularse en procesos investigativos, son las razones que sustentan el nivel de satisfacción obtenido al finalizar la experiencia de movilidad.

Finalmente, los procesos de movilidad deben capitalizarse como una oportunidad para alcanzar el posicionamiento internacional de las universidades. Las IES deben trabajar para que las referencias dadas por los actores que viven la experiencia sean muy favorables, en este caso el total de los jóvenes colombianos y mexicanos afirman estar dispuestos a recomendar a sus compañeros realizar este proceso en las universidades que visitaron.

Ahora bien, estos hallazgos no podrían generalizarse, puesto que se trata de dos universidades públicas que no forman parte del grupo de universidades de élite, cuyo posicionamiento internacional aún carece de notoriedad. Además, se trató de la primera experiencia de un viaje internacional para el total de los jóvenes, donde la novedad constituye un factor favorable para la valoración de la experiencia. A partir de lo encontrado, las futuras líneas de investigación pueden estar relacionadas con estudios en diversos países, donde se incluya el enfoque de los académicos participantes en los procesos de movilidad internacional, así como el enriquecimiento de cada plan de estudios desde las estancias académicas en otros países. Un estudio más general puede estar relacionado con el diagnóstico de la internacionalización del currículum y las opciones de actualización de los planes de estudio con alcance internacional.

\section{Agradecimientos}

Los autores agradecen a la Universidad de los Llanos y a la Universidad Veracruzana por el apoyo brindado en el desarrollo del proceso de movilidad académica internacional.

\section{REFERENCIAS}

Basabe, N.; Zlobina, A. y Páez, D. (2004). Integración socio-cultural y adaptación psicológica de los inmigrantes extranjeros en el País Vasco. Cuadernos Sociológicos Vascos. Vitoria, España: Servicio Central de Publicaciones del Gobierno Vasco.

Bermúdez, R. R. (2015). La movilidad internacional por razones de estudio: Geografía de un fenómeno global. Migraciones internacionales, 8 (1), 95-125. 
Bilecen, B. and Mol, Ch. V. (2017). Introduction: international academic mobility and inequalities. Journal of Ethnic and Migration Studies, 43 (8), 1241-1255.

Brooks, R. and Waters, J. (2011). Student mobilities, migration and the internationalization of higher education. Basingstoke, UK: Edit. Palgrave Macmillan

Castro, S. A. y Lupano, P. M. (2013). Predictores de la adaptación sociocultural de estudiantes universitarios extranjeros en Argentina. Interdisciplinaria, 30 (2), 265-281.

Consejo Nacional de Acreditación (CNA) (2013). Lineamientos para la acreditación de programas de Pregrado. En línea. Recuperado de: https://www.cna.gov.co/1741/ articles-186359_pregrado_2013.pdf

Díaz, B. L.; Torruco, G. U.; Martínez, H. M. y Varela, R. M. (2013). La entrevista, recurso flexible y dinámico. Investigación en Educación Médica, 2 (7), 162-167.

Didou, A. S. (2014). La internacionalización de la educación superior y la ciencia en México, 1993-2013. En Didou, S. y Jaramillo, V. (Eds.). Internacionalización de la educación superior y la ciencia en América Latina: un estado del arte. Caracas, Venezuela: UnesCo-IESLAC.

Didou, A. S. (2016). Geopolítica de la internacionalización universitaria: asignaturas pendientes. Universidades, 69, 4-7.

Dirección General de Relaciones Internacionales (2013). Universidad Veracruzana. En línea. Recuperado de: https://www. uv.mx/internacional/movilidad/

Fernández, R. C.; Carreño, Ch. M.; Cea, V. J.; Santander, A. P. y Yáñez, M. D. (2016). Motivaciones de intercambio en estudiantes universitarios. Revista Global de Negocios, 4 (4), 1-10.
Fernández, L. S.; Fernández, A. S. y Vaquero, G. A. (2007). Proyección internacional de los sistemas de educación superior de América Latina y el Caribe. Revista Iberoamericana para la educación, la ciencia y la cultura, 45, 159-175.

Fernández, L. S.; Pérez, E. C. y Vaquero, G. A. (2009). Movilidad internacional de la universidad española: Análisis regional e institucional del programa Sócrates-Erasmus. Revista de Estudios Regionales, 85, 143-172.

Findlay, A. M.; King, R.; Smith, F. M.; Geddes, A. and Skeldon, R. (2012). World class? An investigation of globalization, difference and international student mobility. Transactions of the Institute of British Geographers, 37 (1), 118-131.

Fiocch, M. C. y Rojas, H. (2015). La experiencia de intercambio estudiantil en el extranjero: análisis de las percepciones de chilenos que en su adolescencia participaron en programas de youth for understanding. Revista Última Década, 43, 207-233.

Fresán, O. M. (2009). Impacto del programa de movilidad académica en la formación integral de los alumnos. Revista de la educación superior, 38 (151), 141-160.

García-Palma, J. J. (2013). Movilidad estudiantil internacional y cooperación educativa en el nivel superior de educación. Revista Iberoamericana de Educación, 61, 59-76.

Geldres, V. V.; Vásquez, F. N. y Ramos, H. R. (2013). Internacionalización de la educación superior en Chile. Movilidad internacional de estudiantes en la Universidad de La Frontera. Revista Iberoamericana de Ciencia, Tecnología y Sociedad, 24 (8), 45-62. 
Hercog, M. and Van de Laar, M. (2017). Motivations and constraints of moving abroad for Indian students. Journal of International Migration and Integration, 18 (3), 749-770.

Huang, Sh. and Yeoh, B. S. A. (2005). Transnational families and their children's education: China's 'study mothers' in Singapore. Global Networks, 5 (4), 379-400.

Instituto Internacional para la Educación Superior en América Latina y el Caribe (IEsalc) (2009). Tendencias mundiales en materia de movilidad estudiantil. En línea. Recuperado de: http:// www.iesalc.unesco.org.ve/index. php?option=com_content\&view $=$ ar ticle\&id=828:tendencias-mundiales-en-materia-de-movilidad-estudiantil\&catid=11\&Itemid=466\&lang $=$ es

Kondakci, Y. (2011). Student mobility reviewed: Attraction and satisfaction of international students in turkey. Higher Education, 62 (5), 573-592.

Lloyd, M. (2016). ¿El gran negocio de la internacionalización de la educación superior? Campus Milenio. En línea. Recuperado de: http://campusmilenio.mx/ index.php?option $=$ com_k2\&view $=\mathrm{i}$ tem\&id=5141:el-gran-negocio-de-la-internacionalizacion-de-la-educacion-superior\&Itemid $=346$

Ministerio de Educación Nacional. (2015). Guías para la internacionalización de educación. Gestión de la internacionalización. En línea. Recuperado de: http://www. colombiaaprende.edu.co/html/directivos/1598/articles-355059_guia_gestion. pdf

Morales, M. J. y Manosalba, T. C. (2016). Dilemas y tensiones alrededor de la Plataforma de Movilidad Académica y
Estudiantil de la Alianza del Pacífico. Universidades, 69, 23-34.

Navarrete, S. B.; Mayor, R. L.; Rangel, C. N. S.; González, G. Z. P.; Zavala, T. V. y Guillén, C. D. (2015). Movilidad estudiantil "Un viaje hacia el aprendizaje". Revista electrónica de investigación en enfermería FESI-UNAM, 4 (8), 17 -24.

Organización de las Naciones Unidas para la Educación, la Ciencia y la Cultura (Unesco) (2010). Internacionalización de la Educación Superior: Nuevos desarrollos y Consecuencias No Intencionadas. En línea. Recuperado de: http://www.iesalc. unesco.org.ve/index.php?option=com_ content\&view=article\&id=2418\%3Ainternacionalizacion-de-la-educacion-superior-nuevos-desarrollos-y-consecuencias-no-intencionadas\&cati$\mathrm{d}=126 \% 3$ Anoticias-pagina-nueva\&Ite$\mathrm{mid}=712$ \&lang=es

Organización de las Naciones Unidas para la Educación, la Ciencia y la Cultura (UnEsco) (2012). Opportunities lost: The impact of grade repetition and early school leaving. En línea. Recuperado de: http:// uis.unesco.org/sites/default/files/documents/opportunities-lost-the-impactof-grade-repetition-and-early-schoolleaving-en_0.pdf

Plan Nacional de Desarrollo (2013). En línea. Recuperado de: http://pnd.gob.mx/ wp-content/uploads/2013/05/PND.pdf

Rincón, M. M. (2013). Movilidad de doctores colombianos. Revisión del estado del arte frente a la internacionalización de la educación superior. Criterio Libre, 11(18), 279-304.

Rienties, B.; Hernández, N. N.; Jindal-Snape, D. and Alcott, P. (2013). The role of cultural background and team divisions in developing social learning relations 
in the classroom. Journal of Studies in International Education, 17 (4), 322-353.

Rodríguez, G. R. (2005). Migración de estudiantes: un aspecto del comercio internacional de servicios de educación superior. Papeles de Población, 11 (44), 221-238.

Trejo, P. A. y Rodrigues, S. J. (2017). Movilidad estudiantil y un acceso a la nacionalidad española diferenciado: el caso de los mexicanos, colombianos y brasileños en Galicia. Foro de Educación, 15 (22), p.p. 1-21.

Tünnermann, B. C. (2011). La educación superior frente a los desafíos contemporáneos. Lección Inaugural del Año Académico 2011. Managua, Nicaragua: Universidad Centroamericana.

Urquía-Grande, E. y Del Campo, C. (2016). Erasmus programme effects: a Spanish case. Papeles de Europa, 29 (2), 94-110. 